\title{
BMJ Open Systematic review protocol examining the effectiveness of hospital clowns for symptom cluster management in paediatrics
}

\author{
Luís Carlos Lopes-Júnior, ${ }^{1}$ Regina Aparecida Garcia Lima, ${ }^{1}$ Karin Olson, ${ }^{2}$ \\ Emiliana Bomfim, ${ }^{3}$ Eliane Tatsch Neves, ${ }^{4}$ Denise Sayuri Calheiros da Silveira, ${ }^{5}$ \\ Michelle Darezzo Rodrigues Nunes, ${ }^{6}$ Lucila Castanheira Nascimento, ${ }^{1}$ \\ Gabriela Pereira-da-Silva ${ }^{1}$
}

To cite: Lopes-Júnior LC, Lima RAG, Olson K, et al. Systematic review protocol examining the effectiveness of hospital clowns for symptom cluster management in paediatrics. BMJ Open 2019;9:e026524. doi:10.1136/ bmjopen-2018-026524

- Prepublication history for this paper is available online. To view these files, please visit the journal online (http://dx.doi. org/10.1136/bmjopen-2018026524).

Received 6 September 2018 Revised 19 November 2018 Accepted 3 December 2018

Check for updates

(C) Author(s) (or their employer(s)) 2019. Re-use permitted under CC BY-NC. No commercial re-use. See rights and permissions. Published by BMJ.

For numbered affiliations see end of article.

Correspondence to

Dr Luís Carlos Lopes-Júnior; luisgen@usp.br

\section{ABSTRACT}

Introduction Clown intervention may playing an important complementary role in paediatric care and recovery. However, data on its utility for symptom cluster management of hospitalised children and adolescents in acute and chronic disorders are yet to be critically evaluated. As clinicians strive to minimise the psychological burden during hospitalisation, it is important that they are aware of the scientific evidences available regarding clown intervention for symptom management. We aim to provide quality evidence for the effectiveness of clown intervention on symptom cluster management in paediatric inpatients, both in acute and chronic conditions.

Methods and analysis A systematic review of randomised controlled trials (RCTs) and non-randomised controlled trials (NRCTs) will be conducted. MEDLINE, Web of Science, Cochrane Library, Science Direct, PsycINF0, CINAHL, LILACS and SciELO databases will be searched from January 2000 to December 2018. Primary outcomes will include measures related with the effect of clown intervention on symptom cluster of paediatric inpatients (anxiety, depression, pain, fatigue, stress and psychological, emotional responses and perceived wellbeing). Study selection will follow the Preferred Reporting Items for Systematic Reviews and Meta-Analyses guidelines, and the methodological appraisal of the studies will be assessed by the Jadad Scale as well as Cochrane Risk-of-Bias Tool for RCTs, and Risk-of-Bias In NonRandomized Studies Tool for NRCTs. A narrative synthesis will be conducted for all included studies. Also, if sufficient data are available, a meta-analysis will be conducted. The effect sizes will be generated using Hedges' g score for both fixed and random effect models. $I^{2}$ statistics will be used to assess heterogeneity and identify their potential sources.

Ethics and dissemination As it will be a systematic review, without human beings involvement, there will be no requirement for ethical approval. Findings will be disseminated widely through peer-reviewed publication and in various media, for example, conferences, congresses or symposia.

Trial registration number CRD42018107099.
Strengths and limitations of this study

- This protocol reduces the possibility of duplication, gives transparency to the methods and processes that will be used, reduces possible biases and allows peer review.

- This will offer highest level of evidence for informed decisions from this systematic review of randomised controlled trials as well as non-randomised controlled trials.

- This systematic review will be the first to explore the effectiveness of clown intervention for symptom cluster management of hospitalised children and adolescents in acute and chronic disorders.

- The scarcity of of randomised controlled trials undertaken with paediatric inpatients with chronic disorders, the publication bias and the methodological quality of the grey literature found may be the main limitations of the study.

\section{INTRODUCTION}

Illness produces stress, and well-being, self-confidence and psychological processes that may regulate immune responses can be significant factors for recovery and response to treatment. ${ }^{2}$ The procedures and treatments performed in hospital settings can further increase patient burden, especially for hospitalised children and adolescents, requiring specific strategies to help them cope with hospitalisation, avoid stress-related disorders and psychoneurological symptom clusters. ${ }^{2-7}$ Therefore, alleviating psychoneurological symptom clusters caused by the hospitalisation process has become a major interest in paediatric wards. ${ }^{8-17}$ Since therapeutic clowning began in North America in 1986, it has become a popular practice in paediatric settings, mainly in acute and rehabilitation hospitals worldwide. ${ }^{18} 19$ As clown 
intervention, a non-pharmacological approach, has been shown to have a generally positive effect in the outcomes of paediatric patients, ${ }^{18-20}$ reviews conducted on this theme showed conflicting results. ${ }^{21-23}$

It has been shown that this intervention can enhance emotional and behavioural processes, for instance, improving well-being and self-confidence, and reducing stress and anxiety levels. ${ }^{24-32}$ In addition, evidence suggests that hospital clowns help paediatric patients to better adapt to their hospital surroundings and can distract from, and demystify painful or frightening procedures through 'doses of fun' to complement traditional clinical interventions. ${ }^{18} 2730$ This hypothesis is supported by studies showing that clown intervention enhances emotional and behavioural responses. ${ }^{25}{ }^{26}$ Positive changes in emotional responses arising from humour and laughter have been correlated with increased pain thresholds and immunity, inversely correlated with stress hormone levels, and linked to positive health. ${ }^{25} 26$ Despite this recognition, few studies have investigated the molecular mechanisms that mediate the positive health outcomes of clown intervention. ${ }^{33-36}$

Recently, a review of literature has investigated evidences from the 28 randomised controlled trials (RCTs) for the effects of healthcare clowning on children. This review revealed different settings in which RCTs have been conducted such as preoperative areas, during medical procedures and during hospitalisation. Overall, the results show that clown interventions are effective in decreasing negative emotions and psychological symptoms and in enhancing the well-being of patients and their relatives. ${ }^{23}$

Additionally, two systematic reviews and meta-analyses looked at the effects of clown intervention in paediatric hospital settings. ${ }^{21}{ }^{22}$ One of them concluded that hospital clowns play a significant role in reducing stress and anxiety levels in children staying in a paediatric ward or undergoing invasive procedures or minor surgeries under anaesthesia, as well as in their parents, ${ }^{21}$ and the other confirmed the strong effect of clown intervention in reducing children's preoperative psychological distress. ${ }^{22}$ However, both reviews focused solely on acute situations. Furthermore, one of the reviews ${ }^{21}$ looked at both RCTs and non-RCTs (NRCTs), but lacked a specific tool for a bias analysis of the latter. Finally, both failed to investigate the effectiveness of clown intervention for a range of symptom clusters in hospitalised children and adolescents in depth. Hence, in this systematic review we evaluated evidence on the effectiveness of clown intervention for symptom clusters management in hospitalised children and adolescents in a variety of paediatric settings, both in acute and chronic conditions, from both RCTs and NRCTs, assessing the quality of the latter with a recently developed tool, Risk of Bias In Non-randomized Studies of Interventions (ROBINS-1). ${ }^{37}$

This review will expand on the above-mentioned works in order to identify recent methodological and scientific progress until December 2018. Following the Preferred
Reporting Items for Systematic Reviews and Meta-Analyses Protocols (PRISMA-P) checklist as guidance, ${ }^{38}$ we propose a systematic and reproducible strategy to query the literature about the effectiveness of clown intervention on symptom cluster management in paediatric inpatients.

\section{METHODS AND ANALYSIS}

\section{Search strategy}

The search strategy will be performed using resources that enhance methodological transparency and improve the reproducibility of the results and evidence synthesis. In this sense, the search strategy will be elaborated and implemented prior to study selection, according to the PRISMA-P checklist as guidance. ${ }^{38}$ Additionally, using the Population, Intervention, Comparison, Outcome and Study design (PICOS) strategy ${ }^{39}$ we elaborated the guiding question of this review in order to ensure the systematic search of available literature: 'What is the effect of clown intervention for symptom management in hospitalised children and adolescents?' The International Prospective Register of Systematic Reviews registration number is CRD42018107099 (https://www.crd.york.ac.uk/prospero/display_record.php?RecordID=107099).

Studies will be retrieved using eight databases: MEDLINE (via PubMed), Web of Science, Cochrane Library, Science Direct, PsycINFO, CINAHL, LILACS and SciELO. In order to reflect contemporary practice, a search of the literature from the last 18 years (January 2000 to December 2018) will be performed. There will be no restriction regarding the language to avoid the reduce the yield of appropriate articles and also generalisability. In addition, the reference section in the studies returned by the above search was scrutinised for additional relevant articles. It is noteworthy that two researchers (LCLJ and $\mathrm{EOB}$ ) will perform the search strategy independently. Also, the bibliographic software EndNote (https://www. myendnoteweb.com/) will be used to store, organise and manage all the references and ensure a systematic and comprehensive search.

Initially, the existence of controlled descriptors (such as MeSH terms, CINAHL headings, PsycINFO thesaurus and DeCS-Health Science Descriptors) and their synonyms (key words) was verified in each database. The search terms were combined using the Boolean operators 'AND' and 'OR'. ${ }^{40}$

Subsequently, a search strategy combining MeSH terms and free-text words, such as (child OR child, hospitalized OR adolescent OR adolescent, hospitalized OR pediatrics) AND (clown doctors OR medical clown OR clown intervention OR clowns OR therapeutic clown OR clowns in hospital) AND (symptoms OR affective symptoms OR behavioral symptoms OR clusters of neuropsychological symptoms OR neuropsychological symptoms OR anxiety OR stress, psychological OR distress OR psychological impact) was used. In order to locate the clinical trials, we added a filter after the PICOS search strategy 


\begin{tabular}{|c|c|c|}
\hline $\begin{array}{l}\text { PICOS } \\
\text { strategy }^{39}\end{array}$ & Inclusion criteria & $\begin{array}{l}\text { Exclusion } \\
\text { criteria }\end{array}$ \\
\hline P-Population & $\begin{array}{l}\text { Hospitalised children and } \\
\text { adolescents for acute } \\
\text { conditions or chronic } \\
\text { disorders }\end{array}$ & $\begin{array}{l}\text { Non-hospitalised } \\
\text { children and } \\
\text { adolescents }\end{array}$ \\
\hline I-Intervention & Clown intervention & \\
\hline $\begin{array}{l}\mathrm{C}- \\
\text { Comparison }\end{array}$ & $\begin{array}{l}\text { Usual standard of care } \\
\text { without receiving clown } \\
\text { intervention }\end{array}$ & \\
\hline O-Outcome & $\begin{array}{l}\text { Any measure related } \\
\text { to symptom clusters: } \\
\text { anxiety, depression, } \\
\text { pain, fatigue, stress and } \\
\text { psychological, emotional } \\
\text { responses and perceived } \\
\text { well-being }\end{array}$ & $\begin{array}{l}\text { Studies that do } \\
\text { not report any } \\
\text { symptom cluster } \\
\text { as primary } \\
\text { outcome }\end{array}$ \\
\hline $\begin{array}{l}\text { S-Study } \\
\text { design }\end{array}$ & $\begin{array}{l}\text { Randomised controlled } \\
\text { trial and non-randomised } \\
\text { controlled trials (quasi- } \\
\text { experimental study) }\end{array}$ & $\begin{array}{l}\text { All the non- } \\
\text { primary } \\
\text { literature, such } \\
\text { as reviews, } \\
\text { dissertations, } \\
\text { theses, } \\
\text { editorials, } \\
\text { protocol studies } \\
\text { and clinical } \\
\text { guidelines }\end{array}$ \\
\hline
\end{tabular}

that included the following terms: AND (randomized controlled trial OR randomized controlled trials as topic OR controlled clinical trial OR clinical trial OR nonrandomized controlled trials).

\section{Study selection criteria}

A summary of the participants, interventions, comparators and outcomes considered, as well as the type of studies included according to PICOS strategy, is provided in table 1 .

Symptom clusters outcomes will be measured all three dimensions of symptom occurrence, severity and distress. ${ }^{41}$ The key outcome will be measured considering the extent of symptom cluster felt by children during the hospitalisation.

The primary outcome measures will be the number of children with any symptom cluster during hospitalisation, the extent of symptom cluster felt by children measured by any validated scale for the respective symptoms. The secondary outcome measures will be the number of children with acute conditions or chronic disorders, number of children satisfied with the care provided and number of parents satisfied with the care provided.

It is noteworthy that symptom cluster composition, consistency and stability vary widely depending on a host of measurement factors, including the optimal assessment tool (long vs short), the most clinically relevant symptom dimensions (prevalence vs severity or distress caused), the optimal analytical method to derive the cluster, the optimal statistical 'cut-off' points to define symptom cluster and the optimal timing of assessment. ${ }^{41}$ Thus, we will consider in our analysis factors such as variation in measurement timing and the number of symptoms included in an analysis in order to generalisability of symptom cluster over time. ${ }^{42} 43$

\section{Screening and data extraction}

Initial screening of studies will be based on the information contained in their titles and abstracts and will be conducted by two independent investigators (LCLJ and $\mathrm{EOB})$. When the reviewers disagreed, the article will be re-evaluated and, if the disagreement persisted, a third reviewer (ETN) will make a final decision. Full-paper screening will be conducted by the same independent investigators. Cohen's kappa will be used to measure inter-coder agreement in each screening phase.

Data will be extracted using a previously proposed tool, ${ }^{44}$ including four domains: (1) identification of the study (article title; journal title; impact factor of the journal; authors; country of the study; language; publication year; host institution of the study (hospital; university; research centre; single institution; multicentre study)); (2) methodological characteristics (study design; study objective or research question or hypothesis; sample characteristics, eg, sample size, sex; age, race; acute and/or chronic diagnoses; groups and controls; stated length of follow-up; validated measures; statistical analyses, adjustments; (3) main findings and (4) conclusions. If the outcome data in the original article were unclear, the corresponding author will be contacted via email for clarification. For data extraction, two independent Microsoft Excel spreadsheets will elaborated for two reviewers (LCLJ and EOB) to summarise the data from the included studies. Then, the spreadsheets were combined into one. Disagreements will be resolved by a third investigator (ETN).

\section{Quality assessment}

Methodological quality of the RCTs will be assessed using the Jadad Scale, ${ }^{45}$ a widely used tool for classification of the quality of the evidence from RCTs. The Jadad Scale scores range from 0 to 5 , with studies scoring $<3$ considered as low quality and studies that score $\geq 3$ classified as high quality. ${ }^{45}$ The internal validity and risk of bias for RCTs will be assessed with the appraisal tool from the Cochrane Handbook for Systematic Reviews of Interventions V.5.1.0, ${ }^{46}$ which assesses the following study-level aspects: (1) randomisation sequence allocation; (2) allocation concealment; (3) blinding; (4) completeness of outcome data and (5) selective outcome reporting; and classifies studies into low, high or unclear risk of bias. For assessing NRCT, the ROBINS-I, a recently developed tool, will be used. ${ }^{37}$ ROBINS-I is particularly useful to those undertaking systematic reviews that include non-randomised studies of interventions. This tool is guided through seven chronologically arranged bias domains (pre-intervention, at intervention and post-intervention), and the interpretations of domain-level and overall risk of bias judgement 
in ROBINS-I are classified in low, moderate, serious or critical risk of bias. ${ }^{37}$

Two independent reviewers (LCLJ, EOB) will assess the methodological quality of eligible trials. Two independent reviewers will score the selected studies and disagreements will be resolved by a third reviewer (ETN). The risk of bias for each outcome across individual studies will be summarised as a narrative statement, and supported by a risk of bias table. A review-level narrative summary of the risk of bias will also be provided.

\section{Descriptive analysis and meta-analysis}

For studies with a high or unclear risk of bias, defined as high or nuclear risk in $50 \%$ or more of the quality assessment outcomes, a narrative description of the risk of bias will be provided. Risk of bias assessments will be incorporated into synthesis by performing sensitivity analysis (ie, limiting to studies at lowest risk of bias in a secondary analysis).

A narrative synthesis will be conducted for all the included studies. All effect sizes will be transformed into a common metric, in order to make them comparable across studies-the bias-corrected standardised difference in means (Hedges' g) — classified as positive when in favour of the intervention and negative when in favour of the control. For continuous outcome measures, standardised mean differences (SMDs) and risk ratio (RR) for categorical outcomes will be considered for the final assessment from individual studies. SMD was chosen as a measure of pooled results considering the likely variability in the measuring scales for continuous outcomes. ${ }^{21}$ The SMD will be categorised as small, medium and large based on the thresholds $0.2,0.5$ and 0.8 , respectively, as suggested by Cohen's. ${ }^{47}$ The $95 \%$ CI will be used to represent the deviation from the point estimate for both the individual studies and the pooled estimate. Heterogeneity between the studies will be assessed using forest plot visually, as well as $\mathrm{I}^{2}$ statistics. ${ }^{48}$ Random effect models will be used in case of moderate to severe heterogeneity, otherwise fixed effect models will be generated. In addition, the presence of publication bias will be evaluated by use of a funnel plot and the Duval and Tweedie's trim and fill method. ${ }^{49}$

\section{Patient and public involvement}

Patients were not directly involved in the design of this study. As this is a protocol for a systematic review and no participant recruitment will take place, their involvement on the recruitment and dissemination of findings to participants was not applicable.

\section{Amendments}

Any amendments to this protocol will be documented with reference to saved searches and analysis methods, which will be recorded in bibliographic databases (Ovid), EndNote and Excel templates for data collection and synthesis.

\section{Dissemination}

The results of the review will be disseminated in an open access journal to ensure access for undergraduate and graduate students, researchers, academics and research groups and also will be disseminated in various media, such as: conferences, seminars, congresses or symposia.

\section{DISCUSSION}

One of the strengths of the proposed study is to apply a reproducible and transparent procedure for systematic review of the literature. In this protocol, we clearly describe the types of studies, participants, interventions and outcomes that will be included, as well as the data sources, search strategy, data extraction methods (including quality assessment) and methods of combining data. ${ }^{50}$ By publishing the research protocol, we reinforce the clarity of the strategy and minimise the risk of bias, namely selective outcome reporting. ${ }^{46}$ Second, we will focus solely on the impact of the effectiveness of clown intervention on symptom cluster management in paediatric inpatients. This results shall provide high-level information to inform, support and customise decisions from the clinicians in paediatrics settings.

Potential limitations of this study include the heterogeneity of measures and outcomes evaluated and the potentially reduced number of studies in subgroup analyses, which may negatively influence the statistical power in data synthesis.

As clinicians strive to minimise the psychological burden during the hospitalisation process, they must be aware of the scientific evidence available to help them incorporate appropriate laughter and play to clinical practice. ${ }^{18}$ Children and adolescents who require hospitalisation represent a special challenge for the healthcare system as well as for health professionals both because of the illness itself and because of the treatment process. ${ }^{13}{ }^{35}{ }^{36}$ In addition, hospitalised children and adolescents with acute or chronic disorders are also stressed by the separation from their parents, by the hospital environment, by the fear of painful treatments or by the uncertainty in the treatment outcome. ${ }^{20}$ This review will demonstrate the value of the involvement of the hospital clowns for symptom cluster management in paediatric inpatients.

\section{Author affiliations}

${ }^{1}$ WHO Collaborating Centre for Nursing Research Development. Ribeirão Preto, University of São Paulo at Ribeirão Preto College of Nursing, Ribeirao Preto, Brazil

${ }^{2}$ Faculty of Nursing, University of Alberta, Edmonton, Canada

${ }^{3}$ Department of Medicine, University of Saskatchewan, College of Medicine, Saskatoon, Canada

${ }^{4}$ Nursing Department, Federal University of Santa Maria, Santa Maria, Brazil ${ }^{5}$ University of São Paulo at Ribeirão Preto Medical School, Ribeirao Preto, Brazil

${ }^{6}$ Rio de Janeiro State University, Rio de Janeiro, Brazil

Acknowledgements The authors thank the Coordination of Improvement of Higher Education Personnel (CAPES), Brazil, for supporting this research with regular doctoral scholarship to Luís Carlos Lopes Júnior as well as his Doctoral Fellowship/Internship at the University of Alberta (UofA), Edmonton, Alberta, Canada, through the Doctoral "Sandwich" Program Abroad PDSE/CAPES (Process №: BEX 9321/14-4).

Contributors LCL-J, RAGL and KO conceptualised and designed the protocol, drafted the initial manuscript and reviewed the manuscript. LCL-J, EB and ETN defined the concepts and search items, data extraction process as well as 
methodological appraisal of the studies. DSCdS and MDRN planned the data extraction and statistical analysis. LCN and GP-dS, provided critical insights. All authors have approved and contributed to the final written manuscript.

Funding This research was funded by the Coordination for the Improvement of Higher Education Personnel (CAPES), Process number: BEX 9321/14-4

Disclaimer The views of the authors do not necessarily reflect those of the NHS, NIHR or the Department of Health.

Competing interests None declared.

Patient consent for publication Not required.

Provenance and peer review Not commissioned; externally peer reviewed.

Open access This is an open access article distributed in accordance with the Creative Commons Attribution Non Commercial (CC BY-NC 4.0) license, which permits others to distribute, remix, adapt, build upon this work non-commercially, and license their derivative works on different terms, provided the original work is properly cited, appropriate credit is given, any changes made indicated, and the use is non-commercial. See: http://creativecommons.org/licenses/by-nc/4.0/.

\section{REFERENCES}

1. Nassau JH, Tien K, Fritz GK. Review of the literature: integrating psychoneuroimmunology into pediatric chronic illness interventions. J Pediatr Psychol 2008;33:195-207.

2. Caserta MT, O'Connor TG, Wyman PA, et al. The associations between psychosocial stress and the frequency of illness, and innate and adaptive immune function in children. Brain Behav Immun 2008;22:933-40.

3. Lopes-Júnior LC, Bomfim EO, Nascimento LC, et al. Nonpharmacological interventions to manage fatigue and psychological stress in children and adolescents with cancer: an integrative review. Eur J Cancer Care 2016;25:921-35.

4. Nunes MDR, Bomfim E, Olson K, et al. Interventions minimizing fatigue in children/adolescents with cancer: an integrative review. $J$ Child Health Care 2018;22:186-204.

5. Vitorino LM, Lopes-Júnior LC, de Oliveira GH, et al. Spiritual and religious coping and depression among family caregivers of pediatric cancer patients in Latin America. Psychooncology 2018;27:1900-7.

6. Nóia TC, Sant'Ana RSE, Santos A, et al. Coping with the diagnosis and hospitalization of a child with childhood cancer. Invest Educ Enferm 2015;33:465-72

7. Lopes-Júnior LC, Rosa M, Lima RAG. Psychological and psychiatric outcomes following PICU admission: a systematic review of cohort studies. Pediatr Crit Care Med 2018;19:e58-e67.

8. Dodd MJ. Occurrence of symptom clusters. J Natl Cancer Inst Monogr 2004;2004:76-8.

9. Barsevick AM, Whitmer K, Nail LM, et al. Symptom cluster research: conceptual, design, measurement, and analysis issues. J Pain Symptom Manage 2006;31:85-95.

10. Miaskowski C, Aouizerat BE, Dodd M, et al. Conceptual issues in symptom clusters research and their implications for quality-oflife assessment in patients with cancer. J Natl Cancer Inst Monogr 2007;37:39-46.

11. Rodgers CC, Hooke MC, Hockenberry MJ. Symptom clusters in children. Curr Opin Support Palliat Care 2013;7:67-72.

12. Lopes-Júnior LC, Olson K, de Omena Bomfim E, et al. Translational research and symptom management in oncology nursing. Br J Nurs 2016;25:S12-21.

13. Lopes-Júnior LC, de Omena Bomfim E, Nascimento LC, et al. [Theory of unpleasant symptoms: support for the management of symptoms in children and adolescents with cancer]. Rev Gaucha Enferm 2015;36:109-12

14. Nunes MDR, Jacob E, Bomfim EO, et al. Fatigue and health related quality of life in children and adolescents with cancer. Eur J Oncol Nurs 2017;29:39-46.

15. Silva MC, Lopes LC, Nascimento LC, et al. Fatigue in children and adolescents with cancer from the perspective of health professionals. Rev Lat Am Enfermagem 2016;24:e2784.

16. Bomfim E, Anatriello E, Nunes MDR, et al. Correlations between functional Interleukin-1 and changes in fatigue and quality of life in children and adolescents with cancer. $J$ of Clinical Oncology 2015;33:95.

17. Lopes-Júnior LC. Translational research and nursing: the lab bench to bedside. J of Nursing UFPE On Line 2015;9.

18. Spitzer P. Essay: Hospital clowns-modern-day court jesters at work. The Lancet 2006;368:S34-5.
19. Oppenheim D, Simonds C, Hartmann O. Clowning on children's wards. The Lancet 1997;350:1838-40.

20. Koller D, Gryski C. The life threatened child and the life enhancing clown: towards a model of therapeutic clowning. Evid Based Complement Alternat Med 2008;5:17-25.

21. Sridharan K, Sivaramakrishnan G. Therapeutic clowns in pediatrics: a systematic review and meta-analysis of randomized controlled trials. Eur J Pediatr 2016;175:1353-60.

22. Zhang Y, Yang Y, Lau WY, et al. Effectiveness of pre-operative clown intervention on psychological distress: a systematic review and meta-analysis. J Paediatr Child Health 2017;53:237-45.

23. Dionigi A, Dionigi A, Dionigi A. Healthcare clowning: use of specific complementary and alternative medicine for hospitalized children. OBM Integrative and Complementary Medicine 2018;3:1.

24. Bekinschtein TA, Davis $\mathrm{MH}$, Rodd JM, et al. Why clowns taste funny: the relationship between humor and semantic ambiguity. $J$ Neurosci 2011;31:9665-71.

25. Bennett MP, Lengacher C. Humor and laughter may influence health IV. Humor and immune function. Evid Based Complement Alternat Med 2009;6:159-64.

26. Stuber M, Hilber SD, Mintzer LL, et al. Laughter, humor and pain perception in children: a pilot study. Evid Based Complement Alternat Med 2009;6:271-6.

27. Dionigi A, Sangiorgi D, Flangini R. Clown intervention to reduce preoperative anxiety in children and parents: a randomized controlled trial. J Health Psychol 2014;19:369-80.

28. Vagnoli L, Caprilli S, Robiglio A, et al. Clown doctors as a treatment for preoperative anxiety in children: a randomized, prospective study. Pediatrics 2005;116:e563-7.

29. Vagnoli L, Caprilli S, Messeri A. Parental presence, clowns or sedative premedication to treat preoperative anxiety in children: what could be the most promising option? Paediatr Anaesth 2010;20:937-43.

30. Dionigi A, Gremigni P. A combined intervention of art therapy and clown visits to reduce preoperative anxiety in children. J Clin Nurs 2017;26:632-40.

31. Bertini M, Isola E, Paolone G, et al. Clowns benefit children hospitalized for respiratory pathologies. Evid Based Complement Alternat Med 2011;2011:1-9.

32. Meiri N, Ankri A, Hamad-Saied M, et al. The effect of medical clowning on reducing pain, crying, and anxiety in children aged 2-10 years old undergoing venous blood drawing-a randomized controlled study. Eur J Pediatr 2016;175:373-9.

33. Saliba FG, Adiwardana NS, Uehara EU, et al. Salivary cortisol levels: the importance of clown doctors to reduce stress. Pediatr Rep 2016;8:6188

34. Sánchez JC, Echeverri LF, Londoño MJ, et al. effects of a humor therapy program on stress levels in pediatric inpatients. Hosp Pediatr 2017;7:46-53.

35. Lopes-Júnior LC, Pereira-da-Silva G, Silveira DSC, et al. The effect of clown intervention on self-report and biomarker measures of stress and fatigue in pediatric osteosarcoma inpatients: a pilot study. Integr Cancer Ther 2018;17:928-40.

36. Lopes-Júnior LC, Silveira DSC, Olson K, et al. Clown intervention on psychological stress and fatigue in pediatric patients with cancer undergoing chemotherapy. Cancer Nurs 2019;42. Ahead of print.

37. Sterne JA, Hernán MA, Reeves BC, et al. ROBINS-I: a tool for assessing risk of bias in non-randomised studies of interventions. BMJ 2016;355:i4919.

38. Moher D, Shamseer L, Clarke M, et al. Preferred reporting items for systematic review and meta-analysis protocols (PRISMA-P) 2015 statement. Syst Rev 2015;4:1.

39. The Cochrane Collaboration. Chapter 5: Defining the review question and deveoloping criteria for including studies. In: Higgins JPT, Green $\mathrm{S}$, eds. Cochrane handbook of systematic reviews. Version 5.0.1: The Cochrane Collaboration, 2008.

40. Lefebvre C, Manheimer E, Glanville J. Searching for studies. In: Higgins JPT, Greene S, Cochrane handbook for systematic reviews of interventions, Version 5.0. eds, 2008.

41. Dong ST, Butow PN, Costa DS, et al. Symptom clusters in patients with advanced cancer: a systematic review of observational studies. $J$ Pain Symptom Manage 2014;48:411-50.

42. Kim HJ, Abraham I, Malone PS. Analytical methods and issues for symptom cluster research in oncology. Curr Opin Support Palliat Care 2013;7:45-53.

43. Aktas A, Kirkova J, Walsh D, et al. The psychometric properties of cancer multi-symptom assessment instruments: a comprehensive review (310-A). J Pain Symptom Manage 2012;43:334-5. 
44. Carlos L, Cruz LA, Leopoldo VC, et al. Effectiveness of traditional Chinese acupuncture versus sham acupuncture: a systematic review. Rev Lat Am Enfermagem 2016;24:e2762.

45. Jadad AR, Moore RA, Carroll D, et al. Assessing the quality of reports of randomized clinical trials: is blinding necessary? Control Clin Trials 1996;17:1-12.

46. Higgins JPT, Green S. Cochrane handbook for systematic reviews of interventions. 5.1.0 edition. cochrane collaboration. Chichester, UK: John Wiley and Sons, 2016.
47. Cohen J. Statistical power analysis for the behavioral sciences. 2nd edn. Hillsdale, NJ: Lawrence Erlbaum Associates, 1998.

48. Egger M, Davey Smith G, Schneider M, et al. Bias in meta-analysis detected by a simple, graphical test. BMJ 1997:315:629-34.

49. Duval S, Tweedie R. Trim and fill: a simple funnel-plot-based method of testing and adjusting for publication bias in meta-analysis. Biometrics 2000;56:455-63.

50. Silagy CA, Middleton P, Hopewell S. Publishing protocols of systematic reviews: comparing what was done to what was planned. JAMA 2002;287:2831-4. 